\title{
Impact des déversements d'orage sur la qualité de la Seine à l'étiage
}

\author{
Impact of thunderstorm discharges on the quality \\ of the Seine's low-water mark
}

E. Clamagirand

J. Gaillard

\author{
CERGRENE/ENPC \\ 18, avenue de Choisy, 75013 Paris
}

Depuis plusieurs années, un important programme d'études est entrepris dont l'objectif est de décrire et de chiffrer les principales actions d'assainissement et d'épuration à mener pour satisfaire les critères de qualité nécessaires aux usages de l'eau des grandes rivières de l'agglomération parisienne.

La présente communication est une contribution importante à la mise au point d'un modèle de qualité de la Seine devant permettre de définir les ordres de grandeur - et il convient d'insister sur ce terme des rejets tolérables en Seine par temps de pluie, lors du débordement des égoûts unitaires notamment, compatibles avec le respect des critères de qualité souhaités.

For several years now, a major research programme has been going on, the aim of which is to describe and number the main actions to be taken as regards effluent disposal and sewerage purification in order to satisfy the quality criteria required for the uses of water from the major rivers of the Paris region.

The present communication is an important contribution to establishing a quality model of the Seine which will make it possible to define the degrees of magnitude - and it is fitting to use this term - of tolerable rejects into the Seine by rain, especially during unit sewerage overflow, compatible with respect for the desired quality criteria.

\section{Avant Propos}

par A. Lesouef, Chef du Service Qualité des eaux et études, à l'Agence financière de bassin Seine-Normandie.

Depuis plusieurs années, un important programme d'études est entrepris dont l'objectif est de décrire et de chiffrer les principales actions d'assainissement et d'épuration à mener pour satisfaire les critères de qualité nécessaires aux usages de l'eau des grandes rivières de l'agglomération parisienne.

La fraction importante (près de $70 \%$ ) de l'eau potable provenant des rivières Seine, Marne et Oise pour l'alimentation de cette agglomération de plus de dix millions d'habitants justifie clairement les efforts à accomplir pour la maîtrise de la qualité de cette ressource vitale. La protection de la vie piscicole mérite également une attention particulière, certains orages en été ou en automne se traduisant parfois par des mortalités de poissons bien dommageables. Enfin, il ne faut pas oublier que l'agglomération parisienne est responsable pour une grande part de l'état de quasi-anoxie de la Basse-Seine à l'aval de Rouen, ceci étant dû à la nitrification de l'ammonium émis par la région parisienne.

Pour des raisons de complexité croissante, les études ont été menées en deux parties : la première répond à la satisfaction des usages de l'eau pré-cités par temps sec, la seconde au même objectif mais par temps de pluie, les mesures à prendre par temps de pluie s'ajoutant bien sûr à celles nécessaires par temps sec.

La première partie a été achevée grâce notamment à la mise au point et à l'utilisation d'un modèle simple en régime permanent de qualité de la Seine de Montereau à Poses (Lesouef A. et André A., 1982). 
La présente communication est une contribution importante à la mise au point d'un modèle de qualité de la Seine devant permettre de définir les ordres de grandeur - et il convient d'insister sur ce terme - des rejets tolérables en Seine par temps de pluie, lors du débordement des égoûts unitaires notamment, compatibles avec le respect des critères de qualité souhaités.

Le travail effectué a permis de bien établir, parmi plusieurs options possibles, les principales caractéristiques nécessaires d'un modèle adapté à la fois à l'objectif et aux données disponibles. On sait maintenant grâce aux auteurs, E. Clamagirand et J. Gaillard, qu'un tel modèle n'a pas à prendre en compte les phénomènes hydrauliques transitoires résultant des déversements d'orage, ce qui représente une simplification d'un intérêt considérable, et doit prendre en compte au moins la dispersion longitudinale. Une réponse claire à un problème complexe : telle est la vertu d'une analyse pertinente et d'une somme de travail impressionnante.

\section{Introduction}

Les efforts déployés depuis plus de 10 ans en vue de réduire les rejets d'eaux usées dans le milieu naturel, ont conduit à une amélioration notable de la qualité de nombreux cours d'eau. La pollution due aux eaux pluviales et aux déversements d'orage des réseaux unitaires n'en a pris que plus d'importance. L'effet de choc qui en résulte, notamment en zones fortement urbanisées peut provoquer de brutales dégradations de la qualité du milieu, à des niveaux largement inférieurs aux seuils admissibles. Les fortes mortalités de poissons observées à l'aval de Paris à la suite des orages de juin et juillet 1982 en sont la preuve.

De nombreuses recherches sont actuellement en cours dans ce domaine. Elles concernent pour l'essentiel la partie "amont " du problème : évaluation du degré de pollution des eaux de ruissellement, études des écoulements transitoires en réseaux, amélioration de la gestion des réseaux par temps d'orage.

La recherche, dont les premiers résultats sont présentés dans cet article, aborde le problème des relations entre le fonctionnement du réseau et le comportement du milieu récepteur. L'objectif final est la définition du modèle mathématique le mieux adapté à l'étude de ces relations, sur la base duquel pourra être élaboré un plan d'expérimentation cohérent pour l'ensemble du système. L'application du modèle à une zone suffisamment vaste aidera à définir les grandeurs à mesurer, les points de mesures ainsi que les fréquences d'échantillonnage. Les données ainsi recueillies permettront de valider les hypothèses ayant conduit à la construction du modèle qui pourra alors être utilisé comme outil de gestion du milieu naturel (évaluation des charges polluantes admissibles au cours d'un déversement, gestion des effluents unitaires...).

Le choix du modèle le plus adéquat pour répondre à cette question doit résulter d'un compromis entre les objectifs poursuivis, le degré de finesse dans la schématisation du système réel et les données disponibles. Ce dernier point est certainement le plus contraignant puisque les mesures dont on peut disposer actuellement, tant sur la quantité et la qualité des rejets pluviaux, que sur leur impact sur le milieu récepteur, sont très réduites, éparses, peu coordonnées et correspondent rarement aux évènements responsables des chocs les plus importants.

Il convient, tout d'abord, de rappeler que nous ne nous intéressons ici qu'à l'effet de choc de ces déversements, et plus particulièrement aux chutes d'oxygène dissous par- fois importantes que l'on peut observer dans le milieu, dans les heures ou les jours suivant un évènement pluvieux.

Nous n'aborderons pas d'autres types de pollution tels que la pollution par les hydrocarbures et les substances toxiques, la pollution bactériologique, ou la pollution à plus long terme due à l'injection dans la rivière de grandes quantités de sédiments provenant du lessivage des sols, car cela dépasse le cadre de cette étude, et nécessiterait une approche différente. Il sera toutefois possible, dès que la structure du modèle sera arrêtée, d'intégrer à l'étude de nouveaux paramètres de pollution.

L'un des premiers problèmes auquel on est confronté dans la conception d'un tel modèle est celui de la disparité des échelles de temps des phénomènes mis en jeu. En effet, les évènements orageux qui nous intéressent ont une durée de quelques heures tout au plus. En raison de la réponse très rapide du réseau d'assainissement dans ces milieux fortement urbanisés, le temps caractéristique des déversements sera, lui aussi, de l'ordre de l'heure. Par contre, l'échelle de temps des réactions de biodégradation qui vont modifier la qualité du milieu est de l'ordre du jour. Par conséquent, afin de déterminer le choix du modèle de qualité d'eau le plus adapté, il est apparu essentiel de déterminer au préalable le rôle de ces phénomènes transitoires de courte durée, de nature essentiellement hydraulique, sur le comportement à plus long terme de la qualité du milieu.

Cette étude a permis de définir le degré de finesse nécessaire à la description de ces phénomènes hydrauliques, et, par là-même, la complexité du modèle tout entier.

La démarche suivante a donc finalement été retenue :

1) L'étude a été limitée au tronçon de la Seine compris entre le barrage de Port-à-l'Anglais et l'aval du barrage de Suresnes (c'est-à-dire, essentiellement la traversée de Paris intra-muros), tronçon test suffisant pour étudier la validité des diverses hypothèses envisagées.

2) Une étude hydraulique fine a été tout d'abord menée pour étudier les relations entre les caractéristiques des pluies orageuses et leur impact sur le régime hydraulique de la Seine. Seules les pluies tombant sur Paris intra-muros ont été étudiées, et il n'a donc pas été tenu compte des apports de banlieue.

3) Sur la base de ces résultats, plusieurs modèles de qualité d'eau de structure différente ont été comparés, les paramètres les plus importants ont ainsi été mis en évidence, et le modèle définitif élaboré sur cette base. 


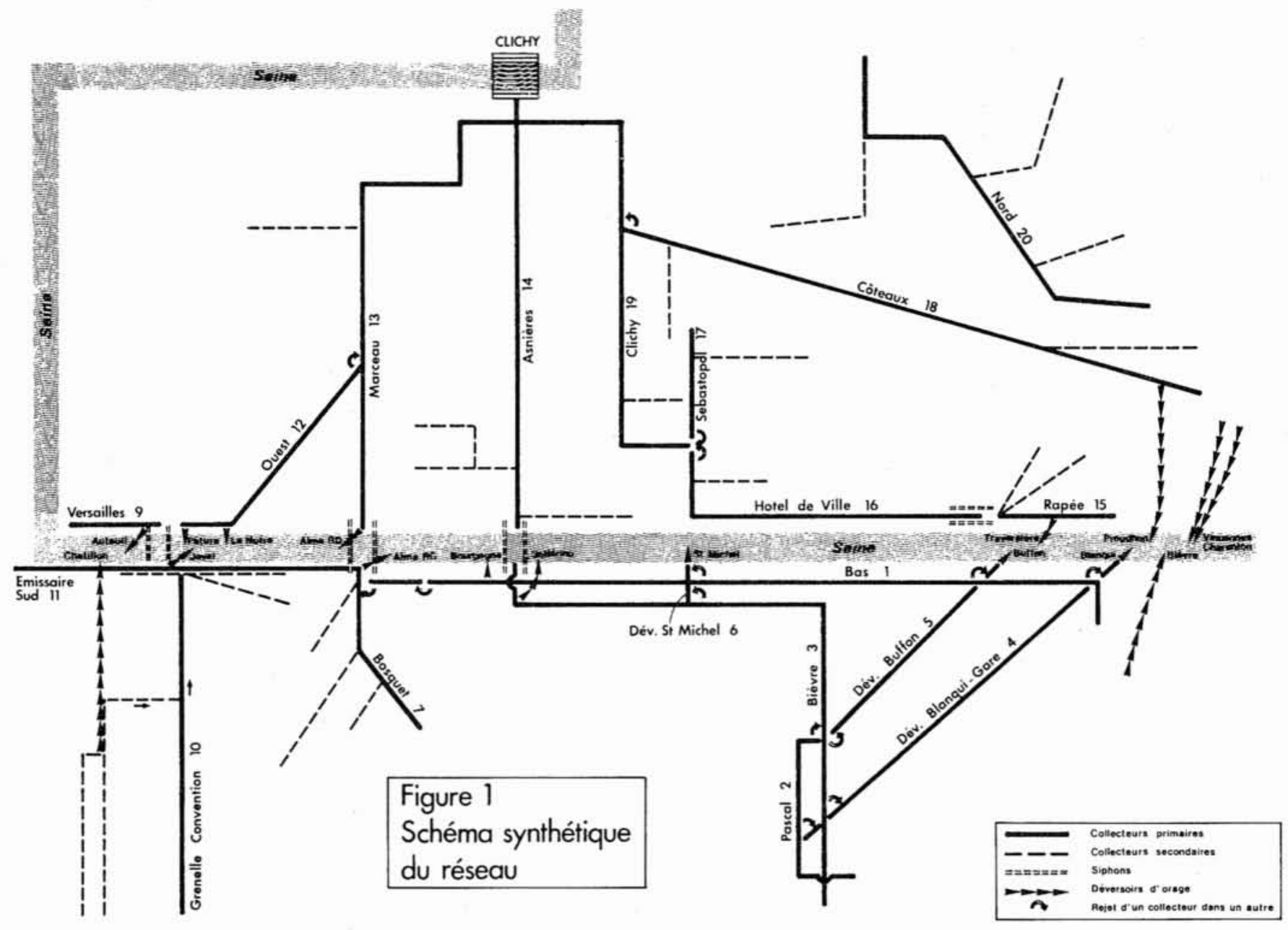

\section{L'étude hydraulique}

L'étude hydraulique a pour but de déterminer aussi finement que possible les perturbations du régime hydraulique de la Seine dues aux déversements d'orage, et notamment les variations des vitesses moyennes des masses d'eau, qui régissent le transport des matières polluantes. Le système étudié se décompose de manière évidente en deux parties distinctes, qui doivent être modélisées séparément : les bassins versants et le réseau d'assainissement (qui transforment la pluie en hydrogrammes de déversements), et l'écoulement en rivière. L'extrême complexité des processus mis en jeu a conduit à faire un certain nombre d'hypothèses simplificatrices, dont les principales sont les suivantes:

- seul le réseau d'assainissement primaire a été modélisé, et les éventuelles mises en charge n'ont pas été prises en compte. Ces deux simplifications ne sont bien sûr pas sans effet sur l'évaluation des temps de transit dans le réseau, et donc sur la forme et la durée des hydrogrammes de déversements, mais une modélisation plus fine du réseau aurait été hors de proportion avec les objectifs relativement globaux de l'étude.
- bien que la dispersion des polluants soit de toute évidence un phénomène tridimensionnel (dispersion transversale et verticale, en plus de longitudinale), le modèle de rivière retenu est un modèle unidimensionnel dans le sens de l'écoulement: les valeurs des variables d'état considérées sont des valeurs moyennes sur une section en travers. Cette simplification est justifiée par le fait que seuls de premiers ordres de grandeurs des phénomènes sont recherchés ici.

\subsection{Le modèle de réseau}

La figure 1 représente la schématisation du réseau d'assainissement primaire de la Ville de Paris, qui a finalement été retenue. Seule a été modélisée la partie du réseau connectée aux déversoirs d'orage de la Ville de Paris. En particulier, une grande partie du réseau situé sur la rive droite de la Seine n'a pas été retenue puisque ses exutoires principaux sont situés vers Clichy, donc à l'aval de Suresnes (limite aval de l'étude hydraulique).

Le modèle retenu est un modèle conceptuel fondé sur un modèle à réservoir linéaire pour la partie ruissellement et sur les méthodes d'onde diffusive ou de translation 

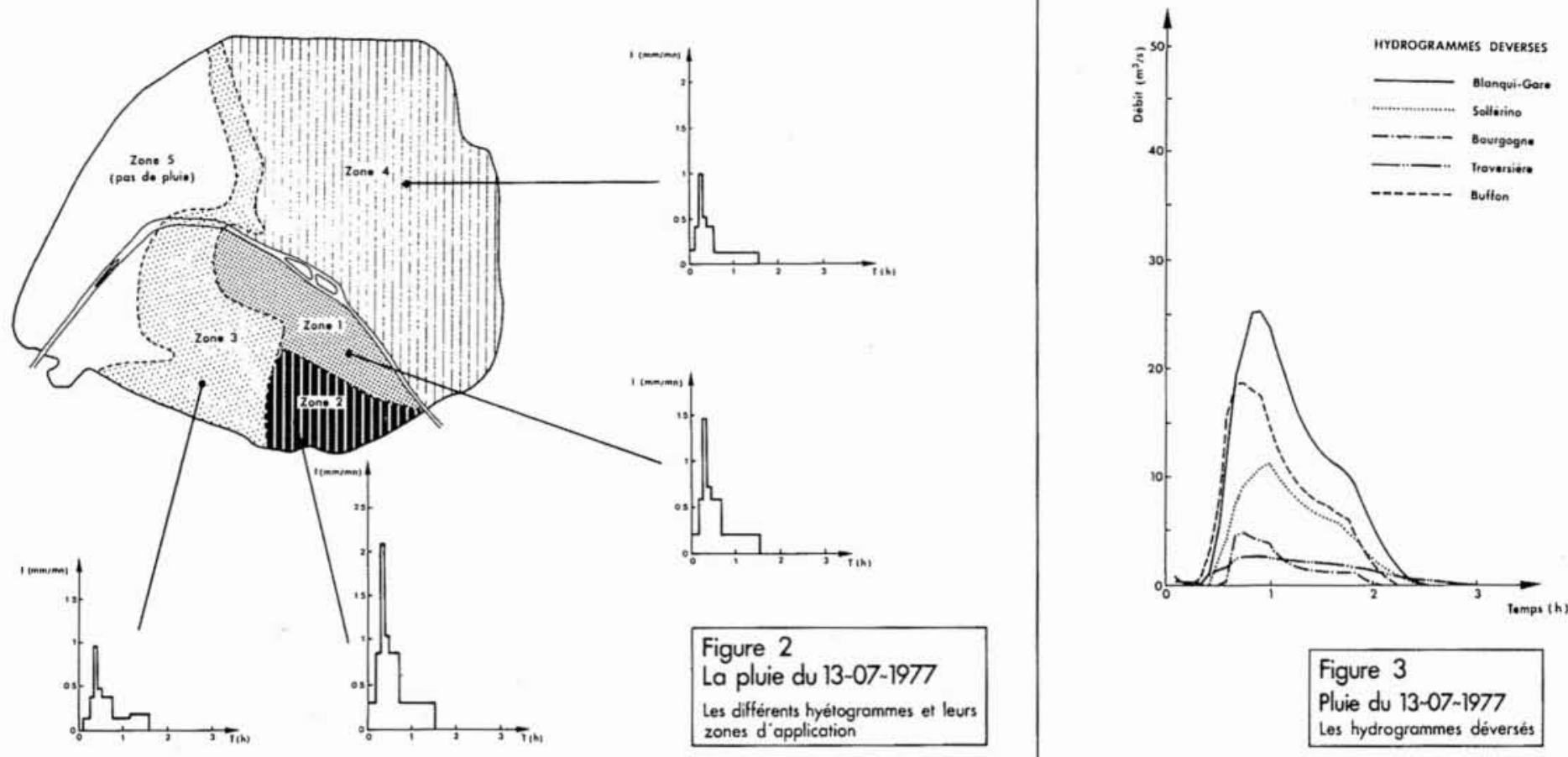

Figure 3

Pluie du 13-07 1977

Les hydrogrammes déversés
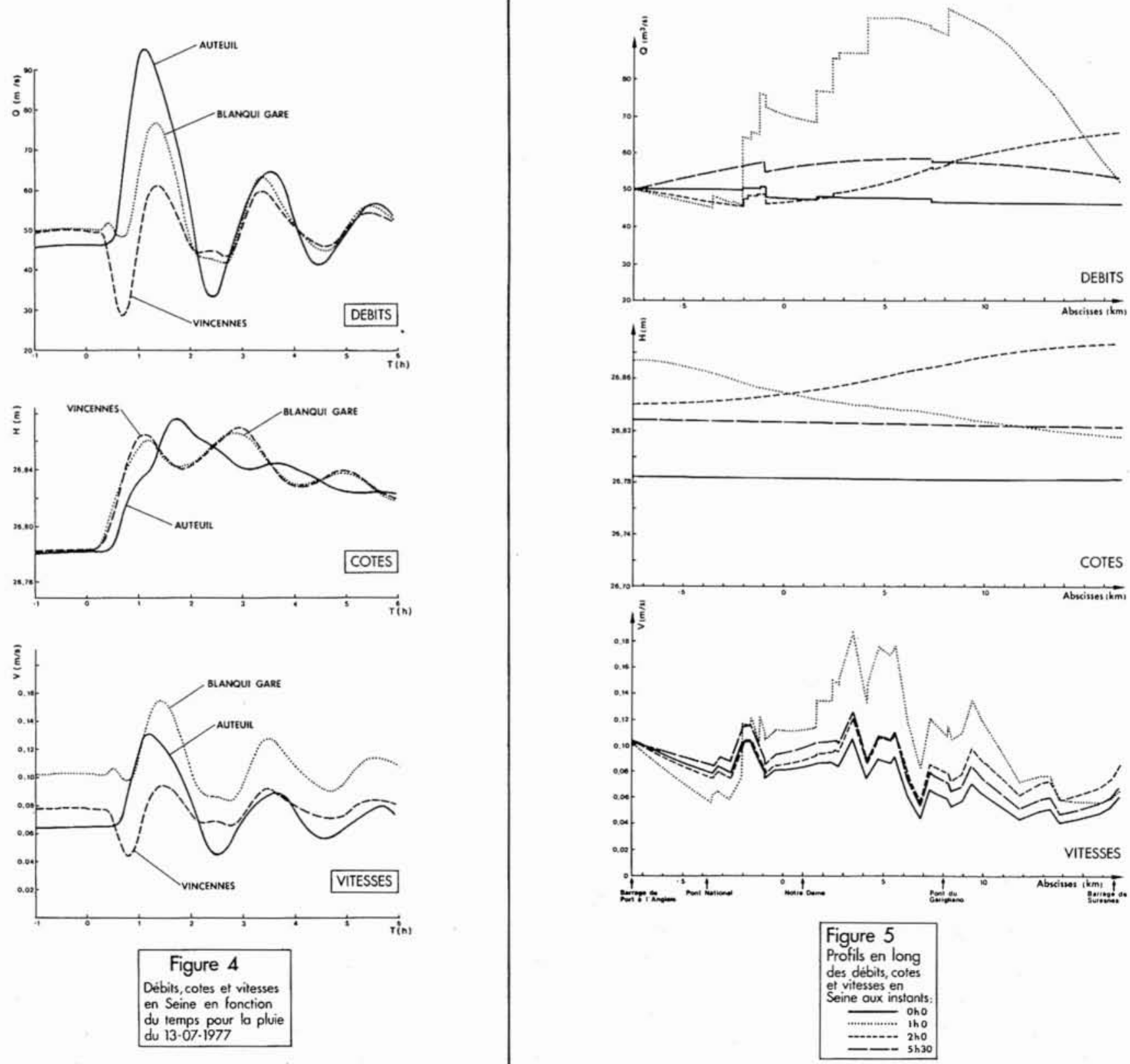
simple pour la partie écoulement en réseau. L'algorithme de base a été développé par l'Agence de bassin SeineNormandie (Bujon, 1983a). Le modèle mis au point dans le cadre de cette étude a été étendu au cas d'un réseau ramifié (les éventuels maillages ont été supprimés) et tient compte, très sommairement cependant, des ouvrages spéciaux. Son application à la Ville de Paris a nécessité un travail de collecte de données entrepris avec la collaboration du Service des Egoûts de la Ville de Paris : limites et caractéristiques générales des bassins versants et sous-bassins versants relatifs aux collecteurs parisiens, caractéristiques des collecteurs.

Les hydrogrammes de déversement sont évalués à partir des hydrogrammes calculés à l'amont immédiat des déversoirs, en supposant que ces derniers écrêtent tous les débits supérieurs à un seuil fixé à 5 fois le débit de temps sec. C'est bien sûr une hypothèse grossière, bien éloignée du comportement véritable d'un déversoir d'orage, mais elle parait toutefois acceptable pour fournir les ordres de grandeur recherchés, surtout dans la gamme de débits déversés très importants dans laquelle se situe l'étude.

Le pas de temps finalement choisi, tant pour la discrétisation de la pluie que pour les calculs du ruissellement et de l'écoulement est de 5 minutes.

\subsection{Le modèle de rivière}

Le modèle uni-dimensionnel de rivière utilisé est le modèle d'écoulement à surface libre en régime transitoire développé par le CEMAGREF (Pochat, 1980). L'hypothèse d'homogénéité transversale est possible pour autant que les débits déversés sont importants par rapport au débit en Seine, ce qui est le cas dans notre étude. Il résoud, par une méthode de résolution implicite, les équations de Saint-Venant, dont il garde tous les termes (termes d'accélération, termes de pression et termes de frottement). Parmi les diverses sorties du modèle, celles que nous avons retenues sont les débits, cotes et vitesses en tous points et à chaque instant.

L'application de ce modèle à la Seine a nécessité que le tronçon étudié (du barrage de Port-à-l'Anglais au barrage de Suresnes, soit $23 \mathrm{kms}$ ), soit divisé en un certain nombre de biefs, à l'amont desquels ont été concentrés tous les apports ponctuels (tels que les déversements d'orage) et les prélèvements (ceux de l'usine Mazas et de l'usine d'Auteuil). Sur les cinquante déversoirs d'orage parisiens, seuls les plus importants ont été retenus, en fonction de l'importance du bassin versant qu'ils drainent et du collecteur sur lequel ils sont situés. Le tronçon d'étude a finalement été découpé en 22 biefs. La condition limite amont est celle d'un débit constant, égal au débit initial (aucune perturbation du système à l'amont de Port-à-l'Anglais); la condition limite aval est celle d'une loi débit-hauteur simulant le fonctionnement du barrage du Suresnes.

Alors que le pas de temps choisi pour modéliser le réseau est de 5 minutes (en raison de l'extrême variabilité des phénomènes pluvieux), il s'est avéré qu'un pas de temps de 15 minutes était suffisant pour modéliser le comportement hydraulique de la rivière. Les hydrogrammes de déversement, déterminés avec un pas de temps de 5 minutes, sont donc moyennés sur 15 minutes avant d'être introduits dans le modèle de rivière.

\subsection{Les résultats}

L'étude a été menée en simulant le comportement du système pour divers événements pluvieux. Six d'entre eux correspondent à des orages historiques, sélectionnés pour leur importance entre 1972 et 1982 . Les autres sont des pluies de projet, élaborées à partir des données statistiques disponibles, selon différentes hypothèses de répartition spatiale.

Nous présenterons ici, pour illustration, l'ensemble des résultats correspondant à l'une des pluies historiques choisies, celle du 13 juillet 1977 (période de retour de 20 ans sur la pluie intense, de 1 à 5 ans sur le reste de l'événement). Le débit initial en Seine est de $50 \mathrm{~m}^{3} / \mathrm{s}$, ce qui correspond à une vitesse initiale moyenne de $0,1 \mathrm{~m} / \mathrm{s}$ et une cote du plan d'eau de $26,78 \mathrm{~m}$. Le coefficient de Strickler a été fixé à 60 (la Seine dans Paris est pratiquement canalisée). Une analyse des données pluviométriques disponible a tout d'abord conduit à diviser la Ville de Paris en 4 zones jugées suffisamment homogènes vis-à-vis de l'événement pluvieux et à chacune desquelles a été affecté un hyétogramme différent (figure 2). Le modèle de réseau fournit ensuite une évaluation des hydrogrammes correspondants en chacun des déversoirs d'orage et dont les plus représentatifs sont présentés en figure 3. Le maximum de débit déversé varie, selon les déversoirs, entre 5 et $15 \mathrm{~m}^{3} / \mathrm{s}$, sauf le déversoir Buffon oủ le maximum atteint presque $20 \mathrm{~m}^{3} / \mathrm{s}$, et le déversoir Blanqui-Gare où il atteint $25 \mathrm{~m}^{3} / \mathrm{s}$. Les valeurs importantes de ces débits laissent supposer qu'en réalité, les collecteurs se mettent en charge, ce qui modifie la forme des hydrogrammes calculés. Toutefois, nous verrons par la suite que ce type d'erreur ne peut modifier significativement les résultats généraux de l'étude.

La figure 4 représente les variations respectives en fonction du temps des débits, des cotes et des vitesses en Seine, en 3 points situés à l'amont immédiat de déversoirs d'orage. La figure 5 présente les profils en long (c'est-à-dire, fonction de l'abscisse comptée dans le sens de l'écoulement) de ces mêmes paramètres à différents instants.

Les principales conclusions que l'on peut retirer de ces résultats sont les suivantes

1) Les déversements créent de très importantes ondes de débits, se propageant soit vers l'aval en provoquant une augmentation des débits et une mise en vitesse des masses d'eau, soit vers l'amont avec stockage d'eau par élévation de la cote de la surface libre et diminution des débits et vitesses. L'amplitude de ces ondes est maximum pendant la durée des déversements (soit approximativement 2 heures dans le cas de la pluie du 13 juillet), mais s'ammortit rapidement après (environ 4 heures après la fin de la pluie du 13 juillet). La célérité de ces ondes peut être très correctement approchée par la formule générale donnant la célérité $C$ d'une intumescence à la surface d'un plan d'eau, soit :

$$
C=\sqrt{g y}
$$

( $g=$ accélération de la pesanteur, $y=$ profondeur de l'eau). Dans le cas étudié, on obtient une célérité de l'ordre de $7 \mathrm{~m} / \mathrm{s}$, à mettre en rapport avec la vitesse moyenne des masses d'eau, soit 10 à $20 \mathrm{~cm} / \mathrm{s}$.

2) Ces ondes provoquent à leur passage une variation 
Figure 6 Pluie du 13-07-1977

Profils en long de DBO5 (mg/l) aux instonts: _ ono

…2 $2 \times 0$

Figure 7 Pluie du 13-07-1977

Profils en long de DBO5 (mg/l) aux instants: __ ono

Figure 8 Pluie du 13-07-1977

Profils en long d'Oxygéne dissous oux instants :

…...12h:

Figure 9 Pluie du 13-07-1977

Protils en long d Oxygène dissous oux instonts

$-\begin{array}{r}0 \\ 2\end{array}$
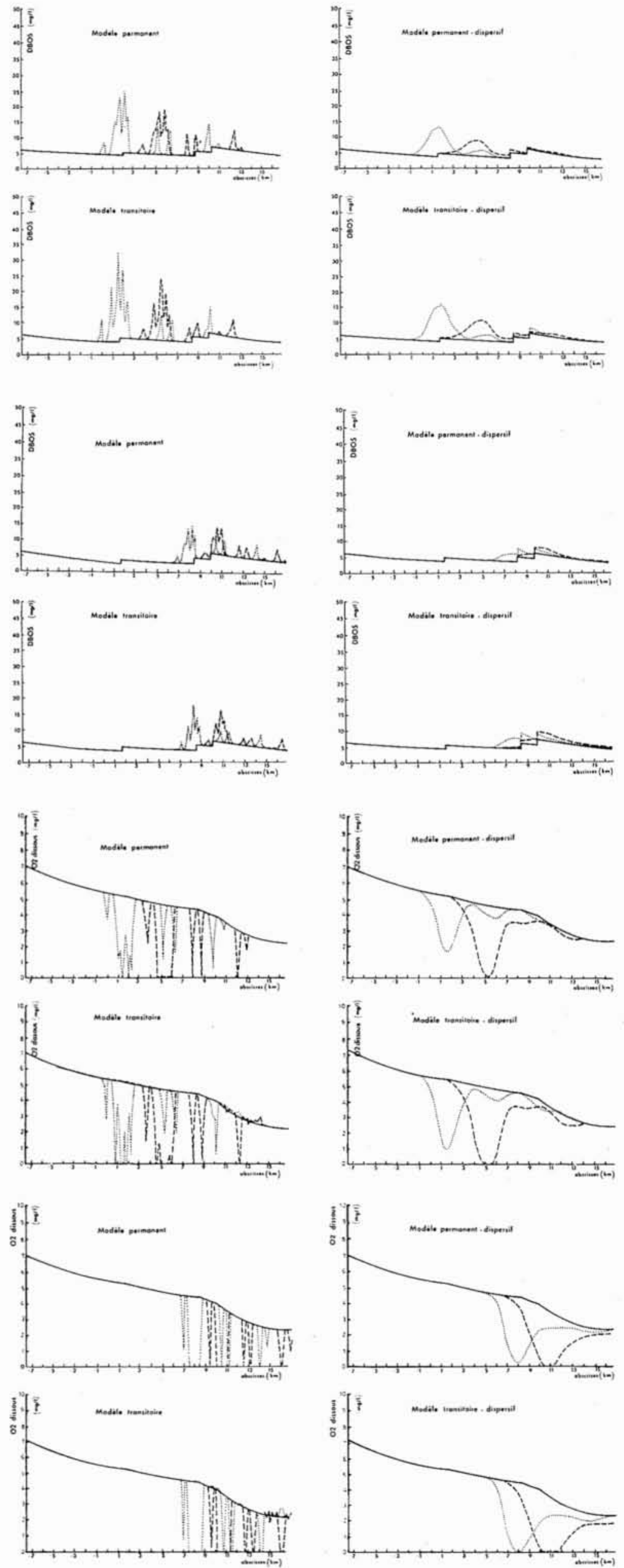
parfois très importante des débits et vitesses, pouvant atteindre $100 \%$ de leurs valeurs initiales.

3) Les cotes de la surface libre varient de manière moins brutale. Le plan d'eau commence à s'élever dans la partie du bief située à l'amont des déversements, l'élévation gagnant progressivement l'aval. Le retour à l'équilibre se fait beaucoup plus progressivement que pour débits et vitesses.

Ces résultats, tirés de l'étude de la pluie du 13 juillet, sont tout à fait représentatifs de ceux obtenus pour l'ensemble des pluies testées. La nature des phénomènes reste inchangée, seuls sont modifiés les ordres de grandeur en fonction de l'importance relative du total des débits déversés par rapport au débit initial en Seine, et de la répartition spatiale des déversements (pour le même débit total déversé, un seul déversement très important engendre des variations sur les grandeurs hydrauliques plus grandes que plusieurs déversements plus faibles).

Il apparaît donc que l'impact hydraulique des déversements sur la rivière peut être extrêmement important en amplitude, mais reste toutefois très limité dans le temps.

\section{Le modèle de qualité d'eau}

\subsection{Le choix du modèle}

Les phénomènes de qualité d'eau auxquels nous nous intéressons ici sont essentiellement de nature physicochimique et leurs échelles de temps sont généralement très supérieures au temps caractéristique des phénomènes hydrauliques (les cinétiques de dégradation sont faibles). Le choix de la structure du modèle définitif repose surtout sur le degré de finesse choisi dans la description des phénomènes hydrauliques: elle sera ainsi très différente selon que l'on travaille en régime permanent ou en régime transitoire. Par contre, une fois cette structure choisie, il est possible de modifier la description initialement retenue des phénomènes de pollution, en rajoutant des variables par exemple, sans pour autant avoir à bouleverser la structure du modèle. C'est pourquoi, nous nous sommes bornés, pour établir le choix du modèle le plus adéquat, à une représentation très simplifiée des processus physico-chimiques, limitée au seul modèle de Streeter et Phelps reliant la demande biochimique en oxygène (DBO) et l'oxygène dissous. Son application à la Seine à Paris a été reprise du travail déjà réalisé par l'Agence de bassin Seine-Normandie (Lesouef et André, 1982). Il sera possible, dans une étape ultérieure, d'affiner la description du système en rajoutant par exemple le cycle de l'azote (Lesouef et André, 1982), sans que les résultats généraux établis ici en soient modifiés.

Le choix du modèle a été effectué en comparant les résultats de 4 modèles différents :

1) Un modèle à régime hydraulique permanent (appelé par la suite "modèle permanent "), dans lequel il n'est pris aucun compte des phénomènes hydrauliques transitoires mis en évidence précédemment, et oủ les déversements d'orage sont assimilés à de simples rejets massiques de polluants sans perturbation des conditions hydrauliques.

2) Un modèle à régime hydraulique transitoire (dit " modèle transitoire "), où les variations des débits, cotes et vitesses en Seine dûes aux déversements d'orage sont prises en compte.

3) Un modèle "permanent-dispersif " identique au modèle permanent mais incluant en plus les phénomènes de dispersion longitudinale.

4) Enfin, un modèle "transitoire-dispersif ", le plus complet, incluant tous les phénomènes hydrauliques dans leur détail, ainsi que la dispersion longitudinale.

Nous ne présenterons pas ici le détail de ces différents modèles et les techniques de résolution numérique employées (E. Clamagirand, 1985), et nous nous bornerons à présenter quelques-uns des résultats les plus significatifs, en particulier ceux obtenus en étudiant l'impact de la pluie du 13 juillet 1977.

Le premier problème à résoudre a été de déterminer les pollutogrammes en chacun des déversoirs d'orage à partir des hydrogrammes de déversement obtenus par le modèle du réseau. Nous avons admis que la concentration des effluents unitaires était constante dans le temps. Ceci suppose que pollutogramme et hydrogramme ont la même forme et conduit à une sous-estimation des charges en début de déversement et une surestimation en fin de déversement. Nous reviendrons ultérieurement sur cette hypothèse simplificatrice.

Les résultats obtenus à partir des 4 modèles considérés, pour la pluie du 13 juillet 1977 , sont résumés sur les figures 6 à 9, qui représentent les profils en long de DB05 et d'oxygène dissous, 12 heures, 24 heures, 36 heures et 48 heures après le début de la pluie (celle-ci ayant duré pendant environ $1 \mathrm{~h} 1 / 2$ ). Pour les deux modèles qui prennent en compte la dispersion, une valeur du coefficient de dispersion d'environ $8 \mathrm{~m}^{2} / \mathrm{s}$ a été retenue conformément aux traçages en Seine réalisés par la Compagnie générale des eaux et à l'exploitation des résultats qui en a été faite par l'Agence de bassin Seine-Normandie (Bujon, 1983b). Nous reviendrons sur ce point au paragraphe suivant.

La comparaison des résultats des différents modèles permet de tirer les conclusions suivantes :

1) Le modèle permanent sous-estime l'amplitude des pics de DB05 par rapport au modèle transitoire. Une étude plus précise a montré que cette différence était dûe à la manière dont est calculé, dans chacun des 2 modèles, le mélange des eaux déversées et des eaux de Seine. Aucun des 2 modèles ne doit en fait représenter la réalité, ces phénomènes de mélange étant trop complexes pour être correctement appréhendés par un modèle uni-dimensionnel quel qu'il soit. Cette différence s'estompe cependant rapidement avec le temps, et son influence sur. l'oxygène dissous reste très limitée.

2) Mis à part ce problème de mélange, les 2 modèles permanent et transitoire donnent des résultats extrêmement voisins. En particulier, quelle que soit l'hypothèse hydraulique faite, les pics de pollution se déplacent pratiquement à la même vitesse, qui est la vitesse moyenne des masses d'eau. En d'autres termes, les phénomènes hydrauliques transitoires consécutifs aux déversements d'orage n'ont pas d'influence significative sur l'évolution de la qualité du cours d'eau.

3) Par contre, les processus de dispersion longitudinale ont une influence capitale sur l'évolution de la pollution. Ils provoquent un "lissage " considérable des phénomènes, avec diminution notable de l'amplitude des pics de DB05 et des déficits d'oxygène dissous. 
Les modèles permanent-dispersif et transitoire-dispersif fournissent des résultats tout-à-fait similaires, mis à part les problèmes de mélange déjà évoqués et dont l'importance reste toutefois très limitée. Le modèle permanent dispersif étant naturellement beaucoup plus facile à mettre en œuvre, pour un temps de calcul très inférieur, il apparait donc qu'un modèle permanent négligeant les phénomènes hydrauliques transitoires dûs au déversement, mais incluant la dispersion longitudinale, résoud le problème en fournissant les ordres de grandeur recherchés avec une précision raisonnable pour un coût minimum.

\subsection{Les paramètres du modèle: Études de sensibilité}

Les trois principaux paramètres du modèle sont

- la constante de biodégradation $K_{1}$

$\left[\frac{\partial L}{\partial t}=-K_{1} L\right.$ ou $L$ représente la DBO et $t$ le temps $]$

- la constante de réaération $K_{2}$

$$
\left[\frac{\partial C}{\partial t}=K_{2}\left(C_{s}-C\right)-K_{1} L\right]
$$

où $C$ représente la concentration en oxygène dissous et $C$, la concentration à saturation,

- et le coefficient de dispersion longitudinale $D$

$\frac{\partial Z}{\partial t}+U \frac{\partial Z}{\partial x}=D \frac{\partial^{2} Z}{\partial x^{2}}+$ Sources-Puits;

$$
\text { avec } Z=C \text { ou } L
$$

Les résultats présentés précédemment ont été obtenus pour les valeurs de ces 3 paramètres déterminés par l'Agence de Bassin Seine-Normandie (Lesouef et André, 1982 et Bujon, 1983b), soit :

$$
\begin{aligned}
K_{1} & =0.6 \text { jour }^{-1} \\
K_{2} & =0.2 \text { jour }^{-1} \\
D & \simeq 8 \mathrm{~m}^{2} / \mathrm{s} .
\end{aligned}
$$

La valeur de $8 \mathrm{~m}^{2} / \mathrm{s}$, retenue pour le coefficient de dispersion, ne doit être considérée que comme une simple approximation, cependant très plausible, du fait qu'elle a été déterminée à partir du calage d'un modèle bidimensionnel et pour un débit sensiblement supérieur à $50 \mathrm{~m}^{3} / \mathrm{s}$.

Des études de sensibilité ont été menées sur chacun de ces paramètres dans le cas d'une pluie d'étude durant 35 minutes, d'intensité maximale $6 \mathrm{~mm} / \mathrm{mn}$, et ne provoquant qu'un seul déversement au déversoir de Blanquigare (dans la partie amont du bief parisien de la Seine).

Il en ressort qu'une erreur de 20 à $25 \%$ sur la valeur de l'un des paramètres (dans une gamme proche de leurs valeurs supposées ci-dessus) conduit à une erreur d'environ $10 \%$ sur les concentrations d'oxygène dissous et de DBO5 en Seine. C'est sans doute la détermination du coefficient de dispersion qui reste le plus problématique, car sa gamme de valeurs possibles est très étendue et sa variation avec le débit doit être prise en compte. Des campagnes de mesures spécifiques pour l'évaluer plus précisément devraient certainement être poursuivies.

\section{Les premiers résultats}

Il n'était pas question, dans l'état actuel de l'étude, d'espérer pouvoir caler et valider correctement les modèles développés en raison des nombreuses incertitudes non encore levées: incertitude sur les pollutogrammes de déversement, non prise en compte des apports de banlieue, erreur sur les profils initiaux en Seine. Seules des campagnes de mesures plus spécifiques permettraient de réaliser une telle validation. Toutefois, à la fin de chaque étape du travail, le réalisme des ordres de grandeur fournis par les modèles utilisés a été vérifié, de telle sorte que le modèle finalement retenu puisse être considéré comme un outil de travail suffisamment vraisemblable pour fournir, d'ores et déjà, un certain nombre de résultats généraux.

\subsection{Influence de la forme des pollutogrammes}

La détermination de la quantité de pollution évacuée vers le milieu naturel à chaque déversoir d'orage reste certainement l'une des tâches les plus lourdes à mener expérimentalement, qu'il convient donc de cerner avec précision.

Deux cas ont ainsi été étudiés pour la pluie du 13 juillet 1977 :

- un pollutogramme proportionnel à l'hydrogramme correspondant (cas déjà étudié précédemment), qui suppose que la concentration $C_{d i r}$ des effluents est constante au cours du temps,

- un pollutogramme évalué de telle sorte que la charge polluante déversée à chaque instant soit constante, et telle que la masse totale déversée pendant la durée de l'événement soit identique. Cette charge polluante $(\mathrm{en} \mathrm{kg} / \mathrm{s})$ est égale au produit de la concentration $C_{d e r}$ par le débit déversé moyen, ou, en d'autres termes, à la masse polluante totale déversée, divisée par la durée de l'événement.

Les résultats de cette comparaison sont présentés sur les figures 10 et 11 . Il en ressort que la différence essentielle entre les 2 simulations réside dans l'évaluation des pics de DBO au début d'événement. Ceci est naturel puisque la forme des 2 pollutogrammes est différente. Toutefois, ces différences s'atténuent très rapidement (moins de 3 heures après le début de la pluie), et n'affectent pratiquement pas les profils d'oxygène dissous. Par conséquent, il apparait qu'à l'échelle à laquelle nous examinons les phénomènes (la Seine sur plusieurs dizaines de kilomètres, pendant plusieurs jours suivant la pluie), seuls sont significatifs pour la détermination des quantités de pollution déversées :

- les temps de début et de fin de déversement,

- la masse polluante totale déversée pendant l'événement.

Ce point pourrait sans doute conduire à une simplification notable des protocoles expérimentaux.

\section{2. Étude de différents scénarios pluvieux}

Neuf pluies de projet aux caractéristiques suivantes ont été étudiées :

- durée totale : 3 heures,

- durée de pluie intense : 30 minutes,

- durée du pic : 5 minutes pour les pluies de période de retour inférieures à 25 ans, 10 minutes sinon,

- instant du pic : 10 minutes après le début de la pluie. 

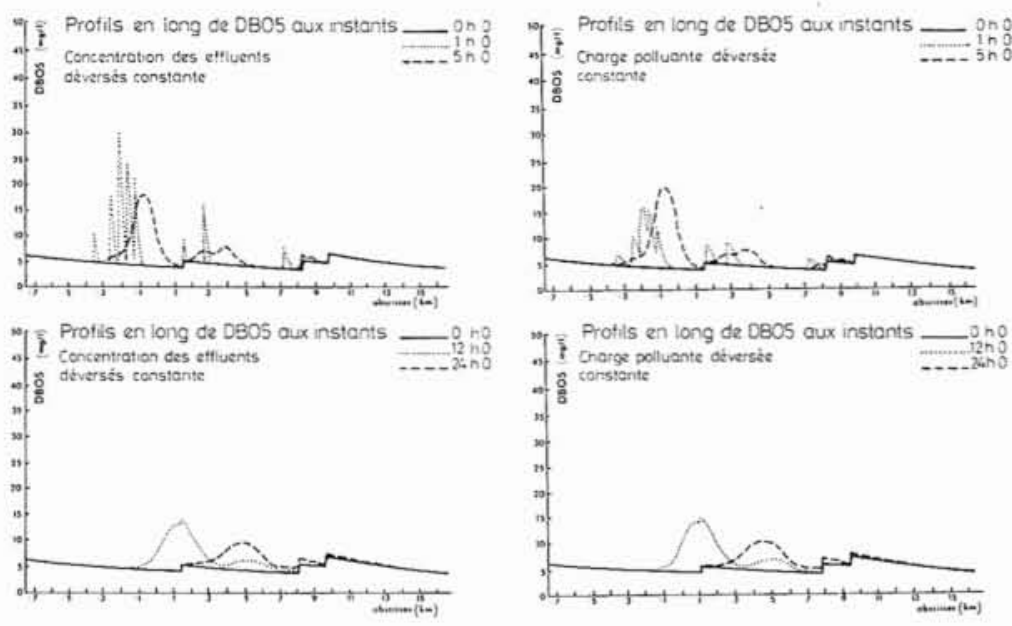

Figure 10 Pluie du 13-07-1977 Influence de la forme du pollutogramme
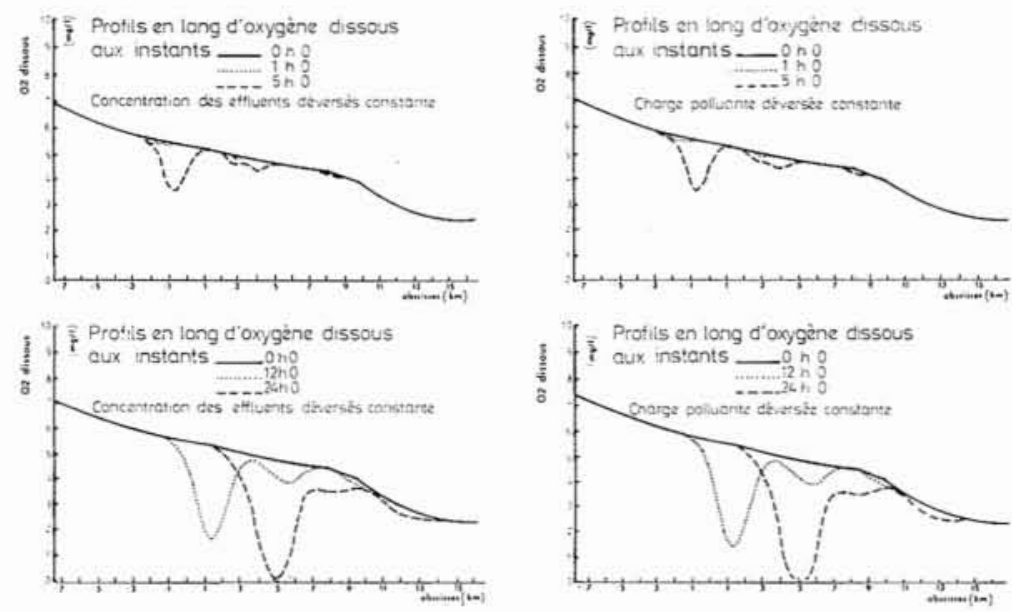

Figure 11 Pluie du 13-07-1977

Influence de la forme du pollutogramme

Les pluies P5, P25 et P100 correspondent à des intensités maximales mesurées à Paris-Montsouris pour des périodes de retour de 5,25 et 100 ans. Elles sont supposées uniformément réparties sur une surface de 720 ha située au sud-est de Paris, et ne sollicitent donc que les déversoirs Blanqui-Gare, Buffon et Saint-Michel.

Les pluies $\mathrm{P}^{\prime} 5, \mathrm{P}^{\prime} 25$. et $\mathrm{P}^{\prime} 100$ correspondent au même volume total d'eau tombée que les précédentes, mais uniformément réparties sur l'ensemble de la Ville de Paris ( 8000 ha). Elles sollicitent donc tous les déversoirs de la Ville de Paris, mais sont beaucoup moins intenses que les précédentes et provoqueront donc des déversements beaucoup plus faibles.

Enfin, les pluies $\Pi 0, \Pi 1$ et $\Pi 2$ sont obtenues en multipliant les intensités de la pluie P100 respectivement par 4/3, 2 et 4 . Les événements $\Pi 0$ et $\Pi 1$ correspondent à des volumes déversés très voisins de ceux des pluies P25 et P100 mais répartis sur tous les déversoirs (et non sur les 3 seuls déversoirs Blanqui-Gare, Buffon et SaintMichel). La pluie $\Pi 2$ représente un événement particulièrement exceptionnel.

Les caractéristiques de chacune de ces 9 pluies sont récapitulées dans le tableau 1.
Les principaux résultats obtenus sont consignés dans les tableaux 2 et 3 correspondant à des débits en Seine respectivement égaux à $50 \mathrm{~m}^{3} / \mathrm{s}$ et $150 \mathrm{~m}^{3} / \mathrm{s}$. Ces tableaux représentent typiquement le genre de résultats que l'on peut attendre de l'utilisation d'un tel modèle. On y notera en particulier que le milieu naturel reste perturbé pendant une à deux semaines par les déversements d'orage et que les zones affectées s'étendent sur plusieurs dizaines de kilomètres, ce qui est parfaitement en accord avec les différentes observations réalisés au cours des dernières années, notamment par le Service de Navigation de la Seine.

\section{Conclusions}

L'objectif de cette étude était de déterminer quels sont les phénomènes les plus importants à prendre en compte pour étudier l'effet de choc produit par les eaux pluviales sur la qualité du milieu récepteur, et d'en déduire la structure du modèle le plus adapté pour en quantifier l'impact. Il en est ressorti que les phénomènes hydrauliques transitoires, consécutifs aux déversements, très importants en 
Tableau 1

Caractéristiques des 9 pluies de projet retenues

\begin{tabular}{|c|c|c|c|c|c|c|}
\hline & $\begin{array}{l}\text { Surface } \\
\text { affectée } \\
\text { (ha) }\end{array}$ & $\begin{array}{l}\text { Volume } \\
\text { d'eau } \\
\left(10^{3} \mathrm{~m}^{3}\right)\end{array}$ & $\begin{array}{l}\text { Lave } \\
\text { d'eau } \\
\text { (mm) }\end{array}$ & $\begin{array}{l}\text { Intensité } \\
\text { sur } \mathrm{s} \text {. } \\
(\mathrm{mm} / \mathrm{mn})\end{array}$ & $\begin{array}{l}\text { Intensité } \\
\text { sur } 30^{\circ} \\
\text { (mm/ma) }\end{array}$ & $\begin{array}{l}\text { Intersité } \\
\text { sur } 3 h \\
(m / m n)\end{array}$ \\
\hline P5 & 720 & 240 & 33 & 2.25 & 0,6 & 0.05 \\
\hline P25 & 720 & 430 & 60 & 2,8 & 1 & 0,13 \\
\hline P100 & 720 & 585 & 81 & 2.7 & 1.3 & 0,20 \\
\hline P.5 & 8000 & 240 & 6 & 0,4 & 0,1 & 0.01 \\
\hline p. 25 & 8000 & 430 & 11 & 0,55 & 0,2 & 0,02 \\
\hline P. 100 & 8000 & 585 & 15 & 0,5 & 0.25 & 0,04 \\
\hline 10 & 8000 & 780 & 20 & 0,7 & 0.35 & 0.05 \\
\hline $\mathrm{n} 1$ & 8000 & 1163 & 30 & 1 & 0,5 & 0,08 \\
\hline 02 & 8000 & 2326 & 60 & 2 & 1 & 0.16 \\
\hline
\end{tabular}

Tableau 2

Caractéristiques de l'évolution des rejets dans le milieu naturel pour les différentes pluies de projet. (Débit initial en Seine : $50 \mathrm{~m}^{3} / \mathrm{s}$.).

\begin{tabular}{|c|c|c|c|c|c|c|c|c|c|}
\hline pture & $\begin{array}{l}\text { VOLUME } \\
\text { DEVERSE } \\
\text { (M3) }\end{array}$ & $\begin{array}{l}\text { CRARGE } \\
\text { DEVERSEE } \\
\text { (TONNES) }\end{array}$ & $\begin{array}{l}\text { PIC DE } \\
\text { DBOS A } \\
12 \mathrm{H} \\
\text { (MG/L) }\end{array}$ & $\begin{array}{l}\text { INSTANT DE } \\
\text { DISPARITIOH } \\
\text { DE LA DBOS } \\
\text { (T) }\end{array}$ & $\begin{array}{l}\text { MININUM D' } \\
\text { OXYGENE } \\
\text { DISSOUS } \\
\text { (MG/L) }\end{array}$ & $\begin{array}{l}\text { INSTANT DO } \\
\text { MINTMUN D } \\
\text { OXYGENE } \\
\text { DISSOUS } \\
\text { (R) }\end{array}$ & $\begin{array}{l}\text { DISTANCE of } \\
\text { LE MINIMUM } \\
\text { D'O2 } \\
\text { EST ATTEINT } \\
\text { (RM) }\end{array}$ & $\begin{array}{l}\text { DUREE DE } \\
\text { LA PERTVR- } \\
\text { BATIOW. } \\
\text { (J) }\end{array}$ & 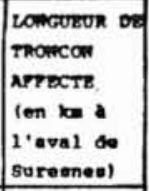 \\
\hline P5 & $15.310^{4}$ & 12.2 & 9.00 & 4.00 & 0.87 & 70.00 & 15.06 & 14.00 & 65.00 \\
\hline P25 & $19.510^{4}$ & 15.6 & 10.20 & 4.00 & 0.59 & 65.00 & 14.05 & 14.00 & 65.00 \\
\hline P100 & $35.9 \quad 10^{4}$ & 28.7 & 14.70 & 4.50 & 0.00 & 20. 75 . & $4.2 \times 15.3$ & 15.00 & $\begin{array}{c}70.00 \\
100 \text { marearux }\end{array}$ \\
\hline P.5 & $2.0 \quad 10^{4}$ & 1.6 & 5.60 & 1.50 & 2.15 & 75. 80. & 16.45 & 6.00 & $\begin{array}{l}15.00 \\
\text { colomber }\end{array}$ \\
\hline$p^{\prime} 25$ & $5.410^{4}$ & 4.3 & 6.81 & 2.00 & 1.93 & 75.00 & 16.45 & 8.00 & $\begin{array}{r}27.00 \\
\text { chatou }\end{array}$ \\
\hline P.100 & $10.510^{4}$ & 8.4 & 6.80 & 3.00 & 1.58 & 70.75. & 16.16 .7 & 10.00 & $\begin{array}{c}40.00 \\
\text { Achires }\end{array}$ \\
\hline$\pi_{0}$ & $18.010^{4}$ & 14.4 & 8.00 & 3.50 & $1 / 03$ & 70.00 & 15.82 & 13.00 & $\begin{array}{l}55.00 \\
\text { Andreny }\end{array}$ \\
\hline$\pi 1$ & $36.210^{4}$ & 29.0 & 10.5 & 4.50 & 0.00 & 55.75. & 14. 16.5 & 15.00 & $\mid \begin{array}{l}70.00 \\
\text { zeo mureaux }\end{array}$ \\
\hline$\pi_{2}$ & $109.0 \quad 10^{4}$ & 80.8 & 16.7 & 5.5 & 0.00 & 15. 85. & 2. 16.8 & 17.00 & $\begin{array}{l}85.00 \\
\text { Manteo 1a } \\
\text { Jolie }\end{array}$ \\
\hline
\end{tabular}


Tableau 3

Caractéristiques de l'évolution des rejets dans le milieu naturel pour les différentes pluies de projet. (Débit initial en Seine: $150 \mathrm{~m}^{3} / \mathrm{s}$ ).

\begin{tabular}{|c|c|c|c|c|c|c|c|c|c|}
\hline PLUIz & $\begin{array}{l}\text { VOLUME } \\
\text { DEVERSE } \\
\text { (M3) }\end{array}$ & $\begin{array}{l}\text { CHARGE } \\
\text { DEVERSEE } \\
\text { (TONNES) }\end{array}$ & $\begin{array}{l}\text { PIC DE } \\
\text { DBOS A } 12 \mathrm{~B} \\
\text { (MG/L) }\end{array}$ & $\begin{array}{c}\text { IRSTANT DE } \\
\text { DISPARITION } \\
\text { DE LA DBOS } \\
\text { (JOUR) }\end{array}$ & $\begin{array}{l}\text { MINIMUM D } \\
\text { OXYGENE } \\
\text { DISGOUS } \\
\text { (KG/L) }\end{array}$ & $\begin{array}{l}\text { INSTANT DU } \\
\text { MINIMUM D } \\
\text { OXYGENE } \\
\text { (H) }\end{array}$ & $\begin{array}{l}\text { DISTANCE OO } \\
\text { LE NIFINUN } \\
\text { D.OXYGENE } \\
\text { EST ATTETWT } \\
\text { (KM) }\end{array}$ & $\begin{array}{l}\text { DOREE DE } \\
\text { LA RERTUR- } \\
\text { BATIOW. } \\
\text { (J) }\end{array}$ & 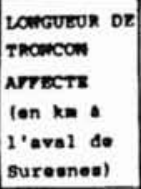 \\
\hline P5 & $15.410^{4}$ & 12.20 & 8.60 & 4.00 & 3.40 & 30.00 & 16.94 & 12.00 & $\begin{array}{l}190.00 \\
\text { Posee }\end{array}$ \\
\hline P25 & $19.510^{4}$ & 15.60 & 9.70 & 4.00 & 2.90 & 30.00 & 16.94 & 12.00 & $\begin{array}{l}190,00 \\
\text { Poues }\end{array}$ \\
\hline P100 & $35.9 \quad 10^{4}$ & 28.70 & 13.00 & 1.50 & 1.30 & 30.00 & 16.94 & 13.00 & 210.00 \\
\hline P.5 & $2.0 \quad 10^{4}$ & 1.60 & 7 & 1.50 & 4.70 & 30.00 & 16.94 & 6.00 & $\begin{array}{r}85.00 \\
\text { Manteo }\end{array}$ \\
\hline D.25 & $5.410^{4}$ & 4.30 & 6.00 & 2.00 & 4.50 & 30.00 & 16.94 & 8.00 & $\begin{array}{l}120.00 \\
\text { Bonniorea }\end{array}$ \\
\hline P'100 & $10.510^{4}$ & 8.40 & 6.50 & 3.00 & 4.30 & 30.00 & 16.94 & 10.00 & $\begin{array}{l}155.00 \\
\text { vernon }\end{array}$ \\
\hline$\pi 0$ & $18.0 \quad 10^{4}$ & 14.40 & 7.50 & 3.50 & 3.90 & 30.00 & 16.94 & 12.00 & $\begin{array}{l}190.00 \\
\text { Poses }\end{array}$ \\
\hline$\pi 1$ & $36.210^{4}$ & 29.00 & 9.50 & 4.50 & 2.94 & 30.00 & 16.94 & 13.00 & 210.00 \\
\hline$\pi_{2}$ & $101.010^{4}$ & 80.8 & 15.00 & 5.00 & 0.00 & 30.00 & 16.94 & 16.00 & 260.00 \\
\hline
\end{tabular}

amplitude, mais de durée très courte, n'ont que peu d'influence sur l'évolution de la qualité du milieu, aux temps de réponse beaucoup plus longs. Par contre, les phénomènes de dispersion longitudinale doivent impérativement être pris en compte. Un modèle hydrauliquement permanent et incluant cette dispersion répond donc à la question.

Bien que la validation d'un tel modèle ne soit actuellement pas possible en raison du peu d'information disponible, il n'en reste pas moins que cet outil permet d'ores et déjà, par l'étude de divers scénarios plausibles et des études de sensibilité, de dégager un certain nombre d'ordres de grandeur utilisables, en particulier, pour mettre sur pied des protocoles expérimentaux adaptés. Il a ainsi été montré qu'une détermination précise des pollutogrammes de déversement n'était sans doute pas nécessaire et que la seule indication de la durée des déversements et de la masse totale de polluant déversé était suffisante.

Une extension de la modélisation à l'ensemble de l'agglomération parisienne devrait sans doute permettre, dans un proche avenir, de définir les phases d'une campagne de mesures qui autorisera la validation du modèle et son utilisation finale pour quantifier l'impact des eaux pluviales sur la Seine à l'étiage et en tirer des consignes de gestion de ces rejets.

\section{Remerciements}

Les auteurs tiennent à remercier ici l'Agence de Bassin Seine-Normandie, et tout particulièrement MM. Lesouef et Bujon, pour l'aide, tant technique que financière, qu'ils leur ont apportée pour la réalisation de ce travail.

\section{Références}

Bujon G., 1983a. - Estimation des débits d'eaux pluviales en milieu urbain - Essai d'application au réseau unitaire de la ville de Paris. AFBSN, 15p.

Bujon G., 1983b. - Modélisation de la dispersion des substances solubles ou pseudo-solubles dans un cours d'eau - Application au cas de la Seine à l'amont de Paris. La Houille Blanche, $\mathrm{n}^{\circ}$ 1/1983, pp. 45-53.

Clamagirand E., 1985. - Modélisation de la qualité des eaux de la Seine à l'étiage en période d'orage. Thèse de DocteurIngénieur, ENPC.

LESOUEF A. et ANDRE A., 1982. - Mise au point d'un modèle de qualité de la Seine de Montereau à Poses. XVII ${ }^{e t}$ journées de l'Hydraulique, Nantes, 14-16 septembre 1982.

Pochat R., 1980. - Ecole d'état de Mécanique des Fluides Hydraulique à surface libre - Hanoï, 283 p. 


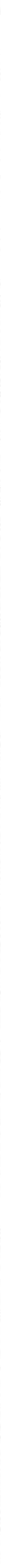

